\title{
Quantum Optics: An Introduction
}

\author{
Min Raj Lamsal \\ Prithwi Narayan Campus, Pokhara \\ Email:min_lamsal@yahoo.com
}

Optics is the physics, which deals with the study of nature of light, its propagation in different media (including vacuum) \& its interaction with different materials. Quantum is a packet of energy absorbed or emitted in the form of tiny packets called quanta. It means the amount of energy released or absorbed in a physical process is always an integral multiple of a discrete unit of energy known as quantum which is also known as photon. Quantum Optics is a field of research in physics, dealing with the application of quantum mechanics to phenomena involving light and its interactions with matter.

Physics in which majority of physical phenomena can be successfully described by using Newton's laws of motion is called classical physics. In fact, the classical physics includes the classical mechanics and the electromagnetic theory. In classical mechanics, the matter is regarded as consisting of point particles which move under the action of mutually interacting forces according to Newton's laws of motion i.e.

$$
\text { Force }(\mathrm{F})=\text { mass }(\mathrm{m}) \times \text { acceleration }(\mathrm{a})
$$

Newton's laws explained successfully the motion of the planets as well as many other electrically neutral macroscopic bodies. The electromagnetic theory explained the physical phenomena concerning electricity and magnetism in terms of electric and magnetic fields. It was Maxwell who unified electric and magnetic fields, called electromagnetic field, and concluded that both electric and magnetic field vectors travel in free space as waves which carry energy. In this way, the classical picture of the world given by the classical physics can be summarized as: the matter consists of point particles carrying mass and radiation consisting of waves carrying energy and momentum.

The laws in classical physics reached its climax in the later half of the nineteenth century. The laws of mechanics started by Newton and their development by Lagrange, Poisson, Jacob, Hamilton etc. seemed perfect and universal. Similarly, Maxwell's equations in electrodynamics gave beautiful and satisfactory description of the electromagnetic phenomena. At the same time, the kinetic theory of gases provided a microscopic basis for the thermo dynamical properties of matter. Up to the end of the nineteenth century, the classical physics was so successful and impressive in explaining physical phenomena that the scientists of that time absolutely believed that they were potentially capable of explaining all physical phenomena.

However, the first indication of inadequacy of the classical physics was seen in the beginning of the twentieth century where it could not explain the experimentally observed spectra of a blackbody radiation. In addition, the laws in classical physics could not explain phenomena involving very high velocities and the phenomena involving very short distance like atoms and other microscopic particles. Similarly, the electromagnetic wave theory could not explain phenomena like Photoelectric effect, Compton Effect etc.

On the way to explain the spectra of blackbody radiation, Max Planck, in 1900, gave birth to a new idea- the idea of quantum energy- that is the energy is absorbed or emitted in the form of tiny packets called quanta. A packet is called a quantum. Planck arrived at the quantum hypothesis by assuming that a simple harmonic oscillator acts as a resonator to radiation. And Planck's idea of quantum is the seed which has grown up as quantum physics in present day physics called modern physics.

Since its birth Max Planck's idea of quantum energy has been in intensive use in explaining various physical phenomena. For example: Einstein in 1904 used this idea to explain photoelectric effect. Similarly, De Broglie in 1912, Bohr \& Frank Hertz in 1913, Stern-Gerlach in 1922, \& Compton in 1923 $\&$ so on used their idea in their theories. And now a day, the quantum physics has become indispensable 
for researches in fundamental physics. While the quantum theory of Planck, as extended to atomic structure by Bohr, Sommerfeld, \& others, has proved to be of inestimable value in the analysis of atomic spectra, other methods of representing atomic processes have been developed which are in still better agreement with the experimental facts.

In the year 1923 De Broglie put forward a new theory of quantum dynamics which at the outset met with little success. At about the same time Heisenberg put forward an independent theory of quantum dynamics which was so successful in its general applications that it attracted wide attention. While both of these theories are different in mathematical formulation Schrodinger was able to show that they lead to the same result $\&$ that the two theories are in reality the same. While quantum mechanics has proved to be so successful in its mathematical formulations of atomic processes that it replaces the older quantum theory.

Quantum Optics is a field of research in physics, dealing with the application of quantum mechanics to phenomena involving light and its interactions with matter. Quantum optics is a branch of quantum physics that focuses primarily on the behavior of light, or photons. At the level of quantum optics, the behavior of individual photons has a bearing on the out coming light, as opposed to classical optics, which was developed by Sir Isaac Newton. Lasers are one application that has come out of the study of quantum optics. Quantum optics (and quantum physics as a whole) views electromagnetic radiation as traveling in the form of a wave and a particle, a phenomena called wave particle duality. The most common explanation of how this works is that the photons move in a stream of particles, but the overall behavior of those particles are determined by a quantum wave function that determines the probability of the particles being in a given location at a given time.

In the realm of quantum physics, observing something actually influences the physical processes taking place. Light waves act like particles and particles act like waves (called wave particle duality). Matter can go from one spot to another without moving through the intervening space (called quantum tunneling). Information moves instantly across vast distances. In fact, in quantum mechanics we discover that the entire universe is actually a series of probabilities. Fortunately, it breaks down when dealing with large objects, as demonstrated by the Schrödinger s Cat thought experiment.

The theory that light moved in discrete bundles (i.e. photons) was presented in Max Planck's 1900 paper on the ultraviolet catastrophe in black body radiation. In 1905, Einstein expanded on these principles in his explanation of the photoelectric effect to define the photon theory of light. Quantum physics developed through the first half of the twentieth century largely by understanding how photons and matter interacted and inter-related. This was viewed, however, as a study of the matter involved more than the light involved. In 1953, the maser was developed (which emitted coherent microwaves) and in 1960 the laser (which emitted coherent light). As the property of the light involved in these devices became more important, quantum optics began being used as the term for this specialized field of study.

Taking findings from quantum electrodynamics (QED), it is also possible to interpret quantum optics in the form of the creation and annihilation of photons, described by field operators. This approach allows the use of certain statistical approaches that are useful in analyzing the behavior of light, although whether it represents what is physically taking place is a matter of some debate. As an example, Lasers (and masers) are the most obvious application of quantum optics. Light emitted from these devices are in a coherent state, which means the light closely resembles a classical sinusoidal wave. In this coherent state, the quantum mechanical wave function (and thus the quantum mechanical uncertainty) is distributed equally. The light emitted from a laser is, therefore, highly ordered, and generally limited to essentially the same energy state (and thus the same frequency \& wavelength). This changed with the invention of the laser in 1950. Laser science i.e., research into principles, design and application of these devices became an important field, and the quantum mechanics underlying the laser's principles was 
studied now with more emphasis on the properties of light, and the name quantum optics became customary. As laser science needed good theoretical foundations, and also because research into these soon proved very fruitful, interest in quantum optics rose. Following the work of Dirac in quantum field theory, Glauber and Mandel applied quantum theory to the electromagnetic field in the 1950s and 1960s to gain a more detailed understanding of photodetection and the statistics of light (see degree of coherence). This led to the introduction the coherent state as a quantum description of laser light and the realization that only some states of light could not be described with classical waves. In the 1970s, Kimball demonstrated the first source of light which required a quantum description, a single atom that emitted one photon at a time. This was first conclusive evidence that light was made up of photons. Another quantum state of light with certain advantages over any classical state, the squeezed light was soon proposed. At the same time, the development of short and ultrashort laser pulses created by Q switching and modelocking techniques, opened the way to the study of unimaginably fast ("ultrafast") processes. Applications for solid state research (e.g. Raman spectroscopy) were found, and mechanical forces of light on matter were studied. The latter led to levitating and positioning clouds of atoms or even small biological samples in an optical trap or optical tweezers by laser beam. This, along with Doppler cooling was the crucial technology needed to achieve the celebrated Bose-Einstein condensation.

Other remarkable results are the demonstration of quantum entanglement, quantum teleportation and (recently, in 2004) quantum logic gates. The latter are of much interest in quantum information theory, a subject which partly emerged from quantum optics, partly from theoretical computer science. Today's fields of interest among quantum optics researchers include parametric down-conversion, parametric oscillation, even shorter (attosecond) light pulses, use of quantum optics for quantum information, manipulation of single atoms, BoseEinstein condensation, their application, and how to manipulate them (a sub-field often called atom optics), and much more.
According to quantum mechanics, light may be considered not only as an electro-magnetic wave but also as a "stream" of particles called photons which travel with $c$, the vacuum speed of light. These particles should not be considered to be classical billiard balls, but as quantum mechanical particles described by a wavefunction spread over a finite region. Each particle carries one quanta of energy given by the relation,

$$
E=h f
$$

Where $\mathrm{h}$ is Planck's constant and $\mathrm{f}$ is the frequency of the light. The postulation of the quantization of light by Max Planck in 1899 and the discovery of the general validity of this idea in Albert Einstein's 1905 explanation of the photoelectric effect soon led physicists to realize the possibility of population inversion and the possibility of the laser.

A frequently encountered state of the light field is the coherent state as introduced by R. J. Glauber in 1963. This state, which can be used to approximately describe the output of a single-frequency laser well above the laser threshold, exhibits Poissonean photon number statistics. Via certain nonlinear interactions, a coherent state can be transformed into a squeezed coherent state, which can exhibit super- or subPoissonean photon statistics. Such light is called squeezed light. Other important quantum aspects are related to correlations of photon statistics between different beams. For example, parametric nonlinear processes can generate so-called twin beams, where ideally each photon of one beam is associated with a photon in the other beam. Atoms are considered as quantum mechanical oscillators with a discrete energy spectrum with the transitions between the energy eigen states being driven by the absorption or emission of light according to Einstein's theory with the oscillator strength depending on the quantum numbers of the states. For solid state matter one uses the energy band models of solid state physics. This is important as understanding how light is detected (typically by a solid-state device that absorbs it) is crucial for understanding experiments.

Research into quantum optics, which aims to bring photons to use for information transfer and 
computation, is now often called photonics to emphasize the claim that photons and photonics will take the role that electrons and electronics now have. According to quantum mechanics, light may be considered not only as an electro-magnetic wave but also as a "stream" of particles called photons which travel with $c$, the vacuum speed of light. These particles should not be considered to be classical billiard balls, but as quantum mechanical particles described by a wave function spread over a finite region. Each particle carries one quanta of energy equal to $h f$, where $\mathrm{h}$ is Planck's constant and $\mathrm{f}$ is the frequency of the light. The postulation of the quantization of light by Max Planck in 1899 and the discovery of the general validity of this idea in Albert Einstein's 1905 explanation of the photoelectric effect soon led physicists to realize the possibility of population inversion and the possibility of the laser. This kind of use of statistical mechanics is the fundament of most concepts of quantum optics: Light is described in terms of field operators for creation and annihilation of photons i.e. in the language of quantum electrodynamics.

A frequently encountered state of the light field is the coherent state as introduced by R. J. Glauber in 1963. This state, which can be used to approximately describe the output of a single-frequency laser well above the laser threshold, exhibits Poissonean photon number statistics. Via certain nonlinear interactions, a coherent state can be transformed into a squeezed coherent state, which can exhibit super- or subPoissonean photon statistics. Such light is called squeezed light. Other important quantum aspects are related to correlations of photon statistics between different beams. For example, parametric nonlinear processes can generate so-called twin beams, where ideally each photon of one beam is associated with a photon in the other beam.

Atoms are considered as quantum mechanical oscillators with a discrete energy spectrum with the transitions between the energy eigen states being driven by the absorption or emission of light according to Einstein's theory with the oscillator strength depending on the quantum numbers of the states.
Quantum optics is used in different branches of physics like spectroscopy and photonics. Quantum optics is helpful in electron microscopy which is used to have resolution of the order of $20 \mathrm{~A}^{0}$ which is applied in hospitals for surface scanning of burnt layer of skin. It is also used in surface imaging of the materials. The resolution of eye lens is only $0.1 \mathrm{~mm}$ but if transmission electron microscope [TEM] and scanning electron microscope [SEM] are used in TV, monitor then they can have more resolution. Similarly, performance of photoemissive cells, photovoltaic cells, photoconductive cells etc. can be improved by using principles of quantum optics. Similarly, the functioning of Burglar alarms, fire alarms, street light switches and traffic counting devices can be modified efficiently with the help of quantum optics. The principles of quantum optics can also be applied in photography, TV camera, TV receivers, Cinematography, automatic doors, night vision scopes etc. They are also used to measure the transparency or opacity of liquids and solids, to study the spectrum of celestial bodies and their temperature, to control heat in chemical reactors and so on.

Bibliography:

http://gerdbreitenbach.de/gallery

http://www.rpphotonics.com/encyclopedia.html

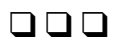

